\title{
Ruminococcus gnavus
}

National Cancer Institute

\section{Source}

National Cancer Institute. Ruminococcus gnavus. NCI Thesaurus. Code C124373.

A species of anaerobic, Gram-positive gut microbes in the class Clostridia. R. gnavus is described as a normal part of the human gut flora, but can form opportunistic infections.

The species is catalase negative, and growth is inhibited by metronidazole, bile, and vancomycin but not by kanamycin or colistin. 